\title{
A systematic review of the effectiveness of discharge care bundles for patients with COPD
}

\author{
Maria B Ospina, ${ }^{1}$ Kelly Mrklas, ${ }^{1,2}$ Lesly Deuchar, ${ }^{1}$ Brian H Rowe, ${ }^{1,3,4}$ Richard Leigh, ${ }^{5}$ \\ Mohit Bhutani, ${ }^{6}$ Michael K Stickland ${ }^{1,6,7}$
}

- Additional material is published online only. To view please visit the journal online (http://dx.doi.org/10.1136/ thoraxjn-2016-208820)

For numbered affiliations see end of article.

\section{Correspondence to} Dr Michael K. Stickland, Associate Professor, Division of Pulmonary Medicine, Department of Medicine University of Alberta, 3-135 Clinical Sciences Building, Edmonton, Alberta, Canada T6G 2J3; michael.stickland@ ualberta.ca

Received 25 April 2016 Revised 28 July 2016 Accepted 13 August 2016 Published Online First 9 September 2016

\section{SLinked}

- http://dx.doi.org/10.1136/ thoraxjnl-2016-208436

- http://dx.doi.org/10.1136/ thoraxjnl-2016-209212

\section{CrossMark}

To cite: Ospina $\mathrm{MB}$ Mrklas K, Deuchar L, et al. Thorax 2017;72:31-39.

\section{ABSTRACT}

Background A COPD discharge bundle is a set of evidence-based practices aimed at improving patient outcomes after discharge from acute care settings following an exacerbation. We conducted a systematic review on the effectiveness of COPD discharge bundles and summarised their individual care elements.

Methods Biomedical electronic databases and clinical trial registries were searched from database inception through April 2016 to identify experimental studies evaluating care bundles offered to patients with COPD at discharge. Random-effects meta-analyses of clinical trials data were conducted for hospital readmissions, mortality, and quality of life (QoL).

Results The review included 14 studies (5 clinical trials, 7 uncontrolled trials, and 2 interrupted time series). A total of 26 distinct elements of care were included in the bundles of individual studies. Evidence from four clinical trials with moderate-to-high risk of bias showed that COPD discharge bundles reduced hospital readmissions (pooled risk ratio (RR): $0.80 ; 95 \% \mathrm{Cl} 0.65$ to 0.99 ).

There was insufficient evidence that care bundles influence long-term mortality (RR: $0.74 ; 95 \% \mathrm{Cl} 0.43$

to 1.28; four trials) or QoL (mean difference in

St. George's Respiratory Questionnaire: 1.84; 95\% Cl -2.13 to 5.8 ).

Conclusions Discharge bundles for patients with COPD led to fewer readmissions but did not significantly improve mortality or QoL. Future studies should employ higher quality research methods, fully report care bundle elements, implementation strategies and intervention fidelity to better evaluate the effectiveness of packaging evidence-based interventions together to improve outcomes of patients with COPD discharged from acute care settings.

\section{INTRODUCTION}

With health improvements leading to longer life expectancy, chronic respiratory diseases have become an increasing health problem and a leading cause of morbidity and mortality worldwide. In particular, COPD places a major burden on health systems ${ }^{1}$ and greatly impacts patients' health status ${ }^{2}$ and quality of life (QoL). ${ }^{3}$ The progressive nature of COPD and predisposition to exacerbations results in frequent healthcare encounters, emergency department (ED) visits and hospital admissions. Optimising care during and after an exacerbation of COPD (AECOPD) is of paramount importance to reduce the risk of relapse and readmission. Despite the large body of evidence

\section{Key messages}

What is the key question?

- In adult patients with an exacerbation of COPD (AECOPD), do discharge care bundles reduce readmissions and improve quality of life?

\section{What is the bottom line?}

- Meta-analysis of randomised controlled trial data shows that discharge care bundles for patients following an AECOPD result in fewer hospital readmissions.

\section{Why read on?}

- This is the first systematic review on COPD discharge care bundles that summarises the current evidence of effectiveness and details the contents of the bundles.

supporting both pharmacological and nonpharmacological interventions to improve health outcomes and reduce future $\mathrm{AECOPD},{ }^{4}$ many patient needs remain unmet. Gaps in the transition from acute to community care have been identified and include lack of access to timely follow-up and disease management programmes, failure to ensure optimal vaccinations, inappropriate medication prescriptions, and failure to address smoking cessation or refer to pulmonary rehabilitation. ${ }^{5}$

Initiatives such as clinical pathways and care bundles aim to meet these challenges and support the translation of evidence-based, effective interventions into daily work to enhance integrated care and optimise patient outcomes. ${ }^{6-8}$ Care bundles have been defined by the Institute for Healthcare Improvement as 'a structured way of improving the processes of care and patient outcomes: a small, straightforward set of evidence-based practicesgenerally three to five-that, when performed collectively and reliably, have been proven to improve patient outcomes". . Care bundles have an important role in quality improvement as they focus on providing consistent and standardised best practice, especially in common chronic conditions (eg, asthma and heart failure). ${ }^{10} 11$

There is evidence that care bundles for admission and in-hospital management of an AECOPD have a positive impact on mortality, hospital readmissions and length of stay. ${ }^{10}{ }^{12}$ Individual evaluations of COPD discharge care bundles have been published 
in the scientific literature; however, no formal synthesis of this body of evidence has been undertaken. Our objective was to systematically review evidence of the effectiveness of COPD care discharge bundles to reduce hospital and ED returns and improve patient-oriented outcomes, and to describe therapeutic components and implementation strategies of care bundles examined in individual studies.

\section{METHODS}

Protocol

This systematic review was conducted and reported in accordance with the Preferred Reporting Items for Systematic Reviews and Meta-Analyses (PRISMA) statement. ${ }^{13}$ The review protocol was registered in the PROSPERO database (CRD42015019333).

\section{Search strategy}

Comprehensive searches of biomedical electronic databases (MEDLINE, EMBASE, CINAHL, Cochrane Central Register of Controlled Trials) and clinical trial registries (Clinicaltrials.org; WHO International Clinical Trials Registry Platform) were conducted from database inception to April 2016. The search strategy was designed by an information specialist and included both selected subject headings and key words related to COPD and discharge care bundles. Details of the search strategy in MEDLINE are presented in online supplementary table S1 (the full search strategy is available upon request). In addition, reference lists of potentially relevant articles were browsed. Primary authors and experts in the area were contacted to identify other potentially relevant studies. No language or publication status restrictions were imposed.

\section{Inclusion and exclusion criteria}

Two reviewers independently screened all titles and abstracts identified by the searches to select potentially relevant articles. Full-text copies of citations deemed relevant, and those whose abstracts and titles provided insufficient information were retrieved for a closer inspection by two independent reviewers who determined study eligibility for the review. Disagreements regarding study selection were resolved through discussions among reviewers.

Randomised controlled trials (RCTs), controlled clinical trials (CCTs), controlled before-and-after (CBA) studies, interrupted time series (ITS) and before-and-after studies (BA) assessing hospital or ED discharge care bundles for patients with AECOPD were included. A discharge care bundle was defined as a set of evidence-based practices that are planned and performed collectively at discharge. ${ }^{11}{ }^{14}$ Primary outcomes of interest were hospital readmissions and ED returns. Secondary outcomes were mortality, physician visits, QoL, patient satisfaction, and economic outcomes.

\section{Risk of bias assessment}

Two independent reviewers assessed the risk of bias of individual studies using standardised instruments based on study design. Discrepancies in risk of bias assessment between reviewers were resolved through discussion. Briefly, RCTs, CCTs, ITS and CBA studies were assessed using standard criteria developed by the Cochrane Effective Practice and Organization of Care (EPOC) group. ${ }^{15}$ Finally, BA studies were assessed with a 12 -item checklist developed by the National Heart Blood and Lung Institute. ${ }^{16}$ An overall assessment of the risk of bias (high, moderate or low) was assigned to individual studies. ${ }^{17}$

\section{Data extraction and synthesis}

Details regarding study characteristics (ie, design, setting, year, country and study duration), participants, intervention, comparison groups and outcomes were obtained from individual studies using a pretested data extraction form and summarised in evidence tables. Characteristics of the COPD discharge care bundles (ie, individual components, providers involved in bundle delivery, implementation strategies and evaluation of implementation fidelity ${ }^{18}$ ) were also obtained. Implementation strategies for discharge care bundles were categorised using the taxonomy developed by the Cochrane EPOC. ${ }^{19}$ Attempts were made to contact authors for important primary data that were missing. All data were extracted by one reviewer and independently verified for accuracy and completeness by a second reviewer. Discrepancies in data extraction were solved through discussion. Study selection, methodological quality assessment and data extraction were managed with Microsoft Excel (Microsoft, Redmond, Washington, USA).

A narrative synthesis of effectiveness outcomes across the studies was undertaken. A 'best-evidence' approach ${ }^{20}$ was adopted to analyse outcome data obtained from a variety of study designs. If possible, outcomes of ITS and BA studies were reported as per cent change relative to baseline and summarised with the median per cent change and IQR. We meta-analysed RCT data using a random-effects model in the absence of clinical and statistical heterogeneity across trials. ${ }^{21}$ Additionally, individual effect sizes against follow-up time for outcome assessment were plotted to assess whether it would be appropriate to combine studies with different follow-up lengths. Pooled risk ratios (RRs) for categorical data and mean differences (MD) with 95\% CIs for continuous outcomes were reported. Forest plots were used to display individual and pooled results. Statistical heterogeneity across trials was assessed using the $\chi^{2}$ test $^{21}$ and quantified with the $\mathrm{I}^{2}$ statistic. Heterogeneity was characterised as small $\left(\mathrm{I}^{2}<25 \%\right)$, moderate $\left(\mathrm{I}^{2}\right.$ between $26 \%$ and $74 \%)$ and high $\left(\mathrm{I}^{2} \geq 75 \%\right){ }^{22}$ In the presence of clinical or statistical heterogeneity, effect sizes with corresponding $95 \% \mathrm{CI}$ were presented separately for each study and sources of heterogeneity were explored qualitatively. A p value $<0.05$ was considered statistically significant in all analyses. Evaluation of publication bias, or the selective publication of research depending on the results, was assessed using funnel plots, ${ }^{23}$ where sufficient data were available from the meta-analyses. Statistical analyses were performed using Review Manager (RevMan) software V.5.3 (Copenhagen: The Nordic Cochrane Centre; The Cochrane Collaboration 2014).

\section{RESULTS}

\section{Search results}

The search strategy (including electronic and manual sources) identified 5863 citations. After removing duplicates and screening 4678 unique titles and abstracts, 113 articles were judged to be potentially relevant, 21 of which satisfied the review eligibility criteria. Of these, 7 references were multiple publications and, therefore, the review included 14 unique studies ${ }^{24-37}$ reported in 21 publications (figure 1). The complete list of excluded studies and reasons for exclusion is available upon request.

\section{Characteristics of studies}

The effectiveness of AECOPD discharge care bundles is a relatively new area of research (median publication year: 2013; IQR: 2012 to 2014); studies were mainly conducted in the 
Figure 1 Preferred Reporting Items for Systematic Reviews and Meta-Analyses (PRISMA) flow chart of study selection for discharge care bundles in patients with an exacerbation of COPD.

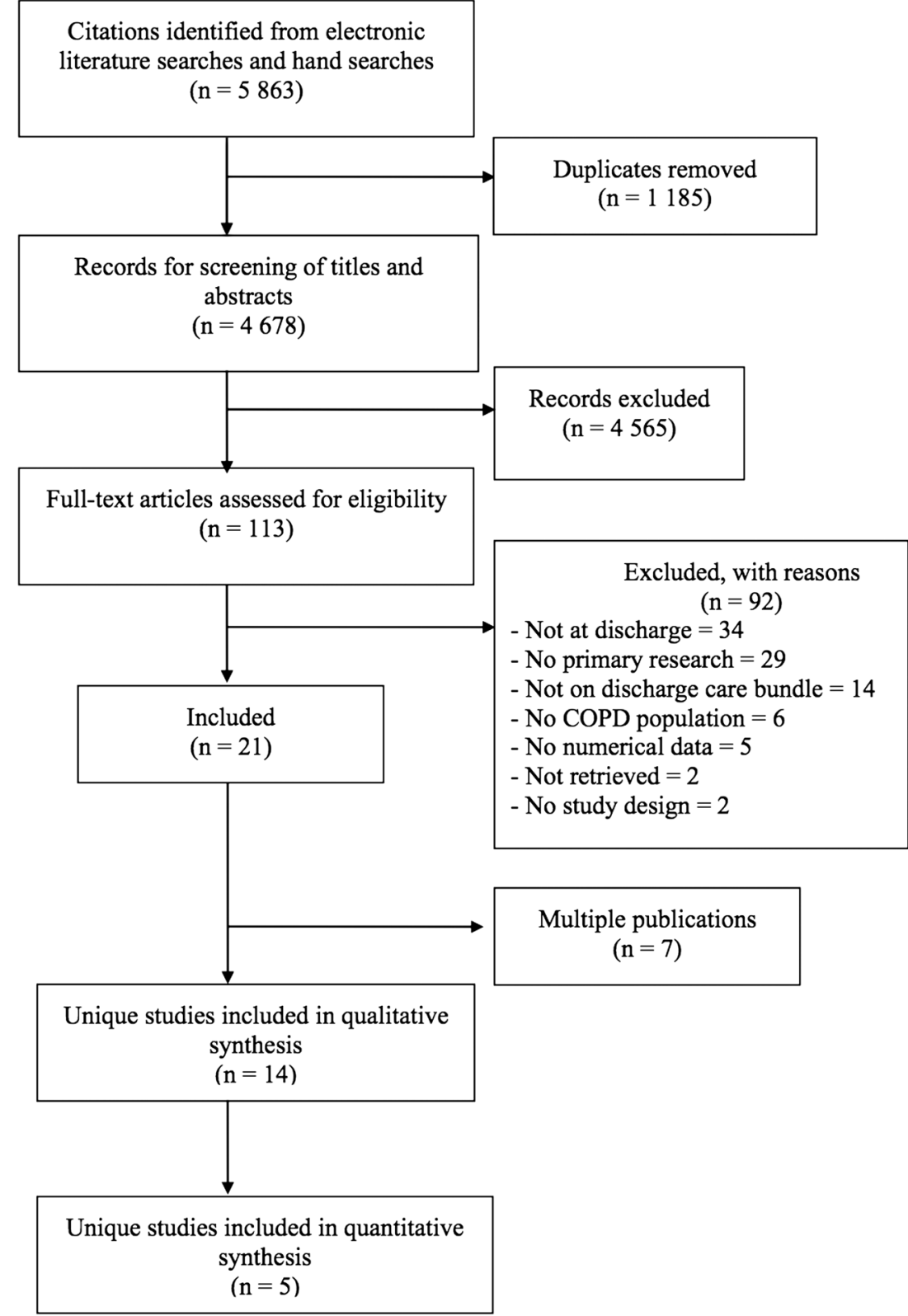

UK $^{26-28} 3133-35$ and the USA ${ }^{29} 3237$ followed by Spain, ${ }^{24} 25$ Canada $^{36}$ and Slovenia. ${ }^{30}$ Apart from four scientific abstracts, ${ }^{26} \quad 27 \quad 35 \quad 37$ all studies were journal publications. Characteristics of individual studies are described in table 1. Of the 14 studies, 4 were RCTs, ${ }^{25} 2930321$ was a CCT, ${ }^{24} 2$ were ITS, ${ }^{26} 31$ and 7 used BA designs. ${ }^{27} 28 \quad 33-37$ Half of the studies published in this area were funded by government, ${ }^{24} 25 \quad 282931-33 \quad 37$ while a few studies received financial support from foundations ${ }^{37}$ or industry partners. ${ }^{36}$ Four studies did not explicitly report the source of funding, ${ }^{26} \quad 27 \quad 34 \quad 35$ whereas one $s+u d y^{30}$ reported that no financial support was received.

All the studies were conducted in acute care settings; however, one study was conducted in a specialised pulmonary hospital. ${ }^{30}$ Most studies were performed in a single centre ${ }^{27-30}$ 33-37 and mainly in academic institutions. ${ }^{24} \begin{array}{llllll}25 & 27 & 28 & 30 & 33-36\end{array}$ More than half of the studies implemented the discharge care bundles in respiratory wards, $\begin{array}{llllllll}24 & 27 & 28 & 30 & 31 & 33-35 & \text { with some }\end{array}$ in medicine/internal medicine wards ${ }^{24} 263136$ or the ED. ${ }^{26}$ Three studies did not report the setting of bundle implementation. $^{25} 2932$ Overall, discharge care bundles were dedicated to patients admitted for AECOPD; however, some studies considered admissions combining COPD and pneumonia $^{3536}$ and COPD and heart failure. ${ }^{32} 36$

\section{Characteristics of the COPD discharge care bundles}

There were important variations in the number and types of interventions incorporated in discharge care bundles across the studies (table 2). Discharge care bundles included between 2 and 12 individual interventions (median: 5; IQR: 4 to 9), and there were 26 distinct elements listed across all bundles. Individual interventions most frequently included in discharge care bundles were: ensuring patients demonstrated adequate inhaler technique (9 studies); educational programme on self-management (9 studies); individually tailored care plans for self-management (8 studies); assessment/referral for pulmonary rehabilitation (8 studies); arrange outpatient follow-up (8 studies); and referral to a smoking cessation programme (7 studies).

Strategies for implementation of discharge care bundles reported in the studies included training of providers, ${ }^{24-26} 28 \quad 32-34$ 
Table 1 Characteristics of individual studies included in systematic review of discharge care bundles administered to patients with AECOPD

\begin{tabular}{|c|c|c|c|c|}
\hline Study; country; design & Intervention, comparison groups (n) & Outcomes and results & $\begin{array}{l}\text { Significant } \\
\text { result }\end{array}$ & $\begin{array}{l}\text { Overall } \\
\text { risk of bias }\end{array}$ \\
\hline $\begin{array}{l}\text { Abad-Corpa et al: }{ }_{i}^{24} \\
\text { Spain; CCT }\end{array}$ & $\begin{array}{l}\text { COPD discharge planning with care bundle } \\
(n=56) \text {, } \\
\text { usual care }(n=87)\end{array}$ & $\begin{array}{l}\text { Hospital re-admissions (\%); f/u: NR } \\
\text { Bundle: } 43 \% \text {; usual care: } 47 \% \text {; NS } \\
\text { Mortality (\%); f/u: NR } \\
\text { Bundle: } 13 \% \text {; usual care: } 20 \% \text {; NS } \\
\text { QoL: SGRQ (mean, SD); f/u: } 3 \mathrm{mo}, 6 \mathrm{mo} \\
\text { Bundle }(3 \mathrm{mo}): 46.5 \pm 22.7 ;(6 \mathrm{mo}): 6 \mathrm{mo}: 46.7 \pm 23.3 \\
\text { Usual care: }(3 \mathrm{mo}): 46.7 \pm 21.9 ;(6 \mathrm{mo}): 44.2 \pm 22.6 ; \mathrm{p}=0.02^{*}\end{array}$ & Yes & High \\
\hline $\begin{array}{l}\text { Casas et al: } \\
\text { Spain, Belgium; RCT }\end{array}$ & $\begin{array}{l}\text { COPD discharge bundle within integrated } \\
\text { care plan }(n=65) \text {, } \\
\text { usual care }(n=90)\end{array}$ & $\begin{array}{l}\text { Hospital re-admissions (\%); f/u: } 1 \mathrm{yr} \\
\text { Bundle: } 23 \% ; \text { usual care: } 29 \% ; p=0.028^{*} \\
\text { Mortality (\%); } 1 \text { yr } \\
\text { Bundle: } 19 \% ; \text { usual care: } 14 \% ; p=0.67 \\
\text { Physician visits: (rate per patient/yr); } 1 \mathrm{yr} \\
\text { Bundle: } 18.4 \% \text {; usual care: } 16.6 \% ; p=0.45\end{array}$ & Yes & Moderate \\
\hline $\begin{array}{l}\text { Jennings et al: }{ }_{i}^{29} \\
\text { USA; RCT }\end{array}$ & $\begin{array}{l}\text { COPD discharge bundle ( } n=93) \text {, } \\
\text { usual care }(n=79)\end{array}$ & $\begin{array}{l}\text { Hospital re-admissions (\%); f/u: } 30 \mathrm{~d} \\
\text { Bundle: } 19.3 \% \text {; usual care: } 22.7 \% ; p=0.58\end{array}$ & No & Moderate \\
\hline $\begin{array}{l}\text { Lainscak et al; } \\
\text { Slovenia; RCT }\end{array}$ & $\begin{array}{l}\text { COPD discharge care bundle }(n=118) \text {, } \\
\text { usual care }(n=135)\end{array}$ & $\begin{array}{l}\text { Hospital re-admissions (\%); f/u: } 6 \text { mo } \\
\text { Bundle: } 31 \% \text {; usual care: } 44 \% ; p=0.033^{*} \\
\text { Mortality (\%); } 6 \text { mo } \\
\text { Bundle: } 9.3 \% \text {; usual care: } 9.6 \% ; p=0.93 \\
\text { QoL: SGRQ (mean, SD); } 6 \text { mo } \\
\text { Bundle: } 55.4(18.8) \\
\text { Usual care: } 53.8(18.6) ; p=0.33\end{array}$ & Yes & Moderate \\
\hline $\begin{array}{l}\text { Linden and Butterworth; } \\
\text { USA; RCT }\end{array}$ & $\begin{array}{l}\text { Components of transitional care model at } \\
\text { discharge }(n=124) \\
\text { usual care }(n=131)\end{array}$ & $\begin{array}{l}\text { Hospital re-admissions (rate per person/time); f/u: } 30 \mathrm{~d}, 90 \mathrm{~d} \\
\text { Bundle }(30 \mathrm{~d}): 0.218 ; \text { usual care }(30 \mathrm{~d}): 0.198 ; p=0.75 \\
\text { Bundle }(90 \mathrm{~d}): 0.50 ; \text { usual care }(90 \mathrm{~d}): 0.55 ; p=0.613 \\
\text { ED returns (rate per person/time); } 30 \mathrm{~d}, 90 \mathrm{~d} \\
\text { Bundle }(30 \mathrm{~d}): 0.129 ; \text { usual care }(30 \mathrm{~d}): 0.16 ; p=0.556 \\
\text { Bundle }(90 \mathrm{~d}): 0.29 ; \text { usual care }(90 \mathrm{~d}): 0.26 ; p=0.761 \\
\text { Mortality }(\%) ; 90 \mathrm{~d} \\
\text { Bundle: } 3.2 \% ; \text { usual care: } 11.5 \% ; p=0.01^{*}\end{array}$ & Yes & Moderate \\
\hline $\begin{array}{l}\text { Graham; } \\
\text { UK; ITS }\end{array}$ & $\begin{array}{l}\text { COPD discharge care planning period }(n=n r), \\
\text { no bundle period (previous year) }(n=n r)\end{array}$ & $\begin{array}{l}\text { Hospital re-admissions (\%); f/u: } \mathrm{nr} \\
\text { Bundle: } 12.8 \% \text {; no bundle: } 14.9 \% ; \mathrm{p}=\mathrm{nr} \text {; per cent change: } \\
-14.10 \% \\
\text { Mortality (\%); f/u: } 1 \mathrm{yr} \\
\text { Bundle: } 6.19 \% ; \text { no bundle: } 4.38 \% ; \mathrm{p}=\mathrm{nr}\end{array}$ & Unclear & High \\
\hline $\begin{array}{l}\text { Laverty et } a l_{i}^{31} \\
\text { UK; ITS }\end{array}$ & $\begin{array}{l}\text { COPD discharge bundle }(n=n r) \text {, } \\
\text { no bundle period (previous year) }(n=n r)\end{array}$ & $\begin{array}{l}\text { Hospital re-admissions (mean annual, SD); f/u: } 28,90 \mathrm{~d} \\
\text { Bundle }(28 \mathrm{~d}):-5.3 \% ; \text { no bundle }(28 \mathrm{~d}): 2.1 \% ; p=0.01 \\
\text { Bundle }(90 \mathrm{~d}):-1.3 \% ; \text { no bundle }(28 \mathrm{~d}): 1.4 \% ; p=0.26\end{array}$ & Yes & High \\
\hline $\begin{array}{l}\text { Halpin et al: }{ }_{i}^{27} \\
\text { UK; BA }\end{array}$ & $\begin{array}{l}\text { COPD discharge care planning period }(n=67) \text {, } \\
\text { no bundle period }(n=257)\end{array}$ & $\begin{array}{l}\text { Hospital re-admissions (\%); f/u: } 30 \mathrm{~d} \\
\text { Bundle: } 18 \% \text {; no bundle: } 27 \% \text {; } p=n \text { r; per cent change: }-33 \%\end{array}$ & Unclear & High \\
\hline $\begin{array}{l}\text { Hopkinson et al: }{ }_{i}^{28} \\
\text { UK; BA }\end{array}$ & $\begin{array}{l}\text { COPD discharge bundle }(n=94) \text {, } \\
\text { no bundle period ( } n=365)\end{array}$ & $\begin{array}{l}\text { Hospital re-admissions (\%); f/u: } 30 \mathrm{~d} \\
\text { Bundle: } 10.8 \% \text {; no bundle: } 16.4 \% \text {; } p=n r \text {; per cent change: }-34.1 \%\end{array}$ & Unclear & High \\
\hline $\begin{array}{l}\text { Mann et ali; } \\
\text { UK; BA }\end{array}$ & $\begin{array}{l}\text { COPD discharge bundle }(n=n r) \text {, } \\
\text { no bundle period }(n=n r)\end{array}$ & $\begin{array}{l}\text { Hospital re-admissions (\%); f/u: } 28 \mathrm{~d} \\
\text { Bundle: } 18 \% \text {; no bundle: } 25 \% ; p=0.06 \text {; per cent change: }-28 \%\end{array}$ & No & High \\
\hline $\begin{array}{l}\text { Matthews et al: } i^{34} \\
\text { UK; BA }\end{array}$ & $\begin{array}{l}\text { COPD discharge bundle }(n=n r) \text {, } \\
\text { no bundle period }(n=n r)\end{array}$ & $\begin{array}{l}\text { Hospital re-admissions (\%); f/u: } 30 \mathrm{~d} \\
\text { Bundle: } 17.7 \% \text {; no bundle: } 23.2 \% \text {; } p=n r \text {; per cent change: }-23.4 \%\end{array}$ & Unclear & High \\
\hline $\begin{array}{l}\text { Seymour et al; }{ }^{35} \\
\text { UK; BA }\end{array}$ & $\begin{array}{l}\text { COPD discharge care planning period } \\
(n=103) \text {, } \\
\text { no bundle period }(n=53)\end{array}$ & $\begin{array}{l}\text { Hospital re-admissions (\%); f/u: } 30 \mathrm{~d}, 3 \mathrm{mo} \\
\text { Bundle }(30 \mathrm{~d}): 17.5 \% \text {; no bundle: } 34 \% ; p=0.02 \text {; per cent change: } \\
-48.5 \% \\
\text { Bundle }(3 \mathrm{mo}): 36.9 \% \text {; no bundle: } 52.8 \% ; p=0.06 \text {; per cent } \\
\text { change: }-30.1 \%\end{array}$ & Yes & High \\
\hline $\begin{array}{l}\text { Shorofsky et al:;6} \\
\text { Canada; BA }\end{array}$ & $\begin{array}{l}\text { COPD discharge bundle }(n=210) \text {, } \\
\text { no bundle period }(n=n r)\end{array}$ & $\begin{array}{l}\text { Hospital re-admissions (\%); f/u: } 90 \mathrm{~d}, 1 \mathrm{yr} \\
\text { Bundle }(90 \mathrm{~d}): 18.6 \% \text {; no bundle: } 32.2 \% ; p=0.017^{*} \text {; per cent } \\
\text { change: } 90 \mathrm{~d}:-42.4 \% \\
\text { Bundle }(1 \mathrm{yr}): 30.5 \% \text {; no bundle: } 61.9 \% ; p=0.038^{*} ; \text { per cent } \\
\text { change: } 1 \mathrm{yr}:-50.7 \%\end{array}$ & Yes & High \\
\hline $\begin{array}{l}\text { Yip et al:;7 } \\
\text { USA; BA }\end{array}$ & $\begin{array}{l}\text { COPD discharge care planning period }(n=n r) \text {, } \\
\text { no bundle period }(n=n r)\end{array}$ & $\begin{array}{l}\text { Hospital re-admissions (\%); f/u: } 30 \mathrm{~d} \\
\text { Bundle: } 21.5 \% \text {; no bundle: } 22.9 \% ; \mathrm{p}=0.1 \text {; per cent change: } \\
-6.11 \%\end{array}$ & No & High \\
\hline
\end{tabular}

*Statistically significant difference.

AECOPD, an exacerbation of COPD; BA, before-and-after study; CCT, controlled clinical trial; d, day(s); ED, emergency department; f/u, follow-up; ITS, interrupted time series; mo, month(s); NR, not reported; NS, not significant; QoL, quality of life; RCT, randomised controlled trial; SGRQ, St. George's Respiratory Questionnaire; yr, year(s).

educational outreach, 242530 distribution of educational materials, ${ }^{25} 2628$ educational meetings, ${ }^{28} 3336$ IT support (web-based service), ${ }^{25}$ audit and feedback, ${ }^{26} 2833$ and continuous quality improvement. $^{28} \quad 33 \quad 34 \quad 36$ Less than half of the studies $^{28} 3133 \quad 34 \quad 36$ had multidisciplinary teams delivering the discharge bundles, and three trials reported that primary care providers (PCP) were involved in bundle implementation. ${ }^{24} 2530$ Evaluation of implementation fidelity was described in five 
Table 2 Individual interventions incorporated in COPD discharge care bundles

\begin{tabular}{|c|c|c|c|c|c|c|c|c|c|c|c|c|c|c|}
\hline Bundle interventions & $\begin{array}{l}\text { Abad-Corpa } \\
\text { et } \mathrm{al}^{24}\end{array}$ & $\begin{array}{l}\text { Casas } \\
\text { et } a l^{25}\end{array}$ & Graham $^{26 *}$ & $\begin{array}{l}\text { Halpin } \\
\text { et } \mathrm{al}^{27}\end{array}$ & $\begin{array}{l}\text { Hopkinson } \\
\text { et } a l^{28}\end{array}$ & $\begin{array}{l}\text { Jennings } \\
\text { et }\left.a\right|^{29}\end{array}$ & $\begin{array}{l}\text { Lainscak } \\
\text { et } \mathrm{al}^{30}\end{array}$ & $\begin{array}{l}\text { Laverty } \\
\text { et }\left.a\right|^{31}\end{array}$ & $\begin{array}{l}\text { Linden and } \\
\text { Butterworth }^{32}\end{array}$ & $\begin{array}{l}\text { Mann } \\
\text { et } a l^{33}\end{array}$ & $\begin{array}{l}\text { Matthews } \\
\text { et } a l^{34}\end{array}$ & $\begin{array}{l}\text { Seymour } \\
\text { et } a l^{35}+\end{array}$ & $\begin{array}{l}\text { Shorofsky } \\
\text { et }\left.a\right|^{36 *}\end{array}$ & $\begin{array}{l}\text { Yip } \\
\text { et } a l^{37}\end{array}$ \\
\hline Medication reconciliation & & & & & & & & & $\sqrt{ }$ & & & & & \\
\hline Prescribe maintenance respiratory medications & & & & & & & & & & & & & $\sqrt{ }$ & $\sqrt{ }$ \\
\hline Provide rescue packs for future exacerbations & & & & & & & & $\sqrt{ }$ & & & $\sqrt{ }$ & & & \\
\hline Comprehensive assessment at discharge & & $\sqrt{ }$ & & & & & & & $\sqrt{ }$ & & & & & \\
\hline Assess suitability for early supported discharge & & & & & & & & & & & $\sqrt{ }$ & & & \\
\hline Individually tailored care plan of self-management & & $\sqrt{ }$ & $\sqrt{ }$ & $\sqrt{ }$ & $\sqrt{ }$ & & & $\sqrt{ }$ & $\sqrt{ }$ & & $\sqrt{ }$ & & $\sqrt{ }$ & \\
\hline $\begin{array}{l}\text { Assessment of patient comprehension of } \\
\text { discharge instructions/use of medications }\end{array}$ & & & & & $\sqrt{ }$ & & & $\sqrt{ }$ & & & & & $\sqrt{ }$ & \\
\hline $\begin{array}{l}\text { Ensure patient has demonstrated adequate inhaler } \\
\text { technique }\end{array}$ & $\sqrt{ }$ & & $\sqrt{ }$ & $\sqrt{ }$ & & $\sqrt{ }$ & $\sqrt{ }$ & $\sqrt{ }$ & & $\sqrt{ }$ & $\sqrt{ }$ & & $\sqrt{ }$ & \\
\hline Educational programme on self-management & & $\sqrt{ }$ & & & $\sqrt{ }$ & $\sqrt{ }$ & $\sqrt{ }$ & $\sqrt{ }$ & $\sqrt{ }$ & $\sqrt{ }$ & $\sqrt{ }$ & & $\sqrt{ }$ & \\
\hline Written information on COPD and medication & & & $\sqrt{ }$ & & $\sqrt{ }$ & & & & & & $\sqrt{ }$ & & & \\
\hline Brief intervention for smoking cessation & & & $\sqrt{ }$ & & & $\sqrt{ }$ & & & & & & & & \\
\hline Referral to smoking cessation programme & & & $\sqrt{ }$ & $\sqrt{ }$ & $\sqrt{ }$ & & & $\sqrt{ }$ & & $\sqrt{ }$ & $\sqrt{ }$ & & $\sqrt{ }$ & \\
\hline Respiratory physiotherapy/breathing exercises & $\sqrt{ }$ & & & & & & $\sqrt{ }$ & & & & & & & \\
\hline Diet recommendations & $\sqrt{ }$ & & & & & & & & & & & & & \\
\hline Hygienic habits recommendations & $\sqrt{ }$ & & & & & & & & & & & & & \\
\hline Adapted exercise & $\sqrt{ }$ & & & & & & & & & & & & & \\
\hline $\begin{array}{l}\text { Screening for gastro-oesophageal reflux disease, } \\
\text { depression or anxiety }\end{array}$ & & & & & & $\sqrt{ }$ & & & & & & & & \\
\hline Home care needs assessment & & & & & & & $\sqrt{ }$ & & & & & & & \\
\hline $\begin{array}{l}\text { Recommend maintenance of home oxygen } \\
\text { therapy apparatus }\end{array}$ & $\sqrt{ }$ & & & & & & & & & & & & & \\
\hline Check $\mathrm{O}_{2}$ saturation on air & & & $\sqrt{ }$ & & & & & & & & & & & \\
\hline Oxygen alert card & & & & & $\sqrt{ }$ & & & $\sqrt{ }$ & & & $\sqrt{ }$ & & & \\
\hline Document spirometry & & & $\sqrt{ }$ & & & & & & & & $\sqrt{ }$ & & $\sqrt{ }$ & \\
\hline Assessment/referral for pulmonary rehabilitation & & & $\sqrt{ }$ & $\sqrt{ }$ & $\sqrt{ }$ & & & $\sqrt{ }$ & $\sqrt{ }$ & & $\sqrt{ }$ & & $\sqrt{ }$ & $\sqrt{ }$ \\
\hline Referral to respiratory specialist nurse & & & $\sqrt{ }$ & & & & & & & & $\sqrt{ }$ & & & \\
\hline Arrange outpatient follow-up & & & $\sqrt{ }$ & $\sqrt{ }$ & $\sqrt{ }$ & & & $\sqrt{ }$ & $\sqrt{ }$ & $\sqrt{ }$ & $\sqrt{ }$ & & $\sqrt{ }$ & \\
\hline Post-discharge phone call & & & & & & $\sqrt{ }$ & & & & & & & & \\
\hline
\end{tabular}

Post-discharge phone call

†Not reported; attempts to obtain information from study authors were unsuccessful. 


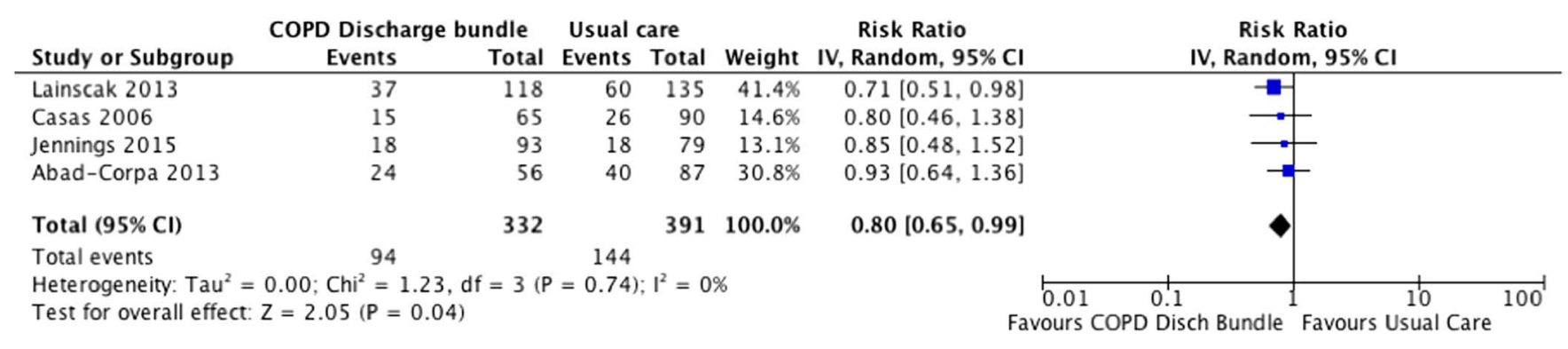

Figure 2 Effectiveness of discharge care bundles on hospital re-admissions for patients with an exacerbation of COPD (randomised controlled trial data only).

studies, ${ }^{26-28} 3233$ all indicating the percentage of patients who actually received individual interventions of the discharge care bundles.

\section{Risk of bias}

Most of the included studies had methodological limitations and the reporting of the risk of bias variables was frequently inadequate. Of the five clinical trials, four ${ }^{25} 293032$ had moderate risk of bias and one ${ }^{24}$ had a high risk of bias. The most common sources of bias in clinical trials related to lack of similarity at baseline and lack of blinding in outcome assessment. Studies with ITS and BA designs demonstrated specific and inherent risk of biases that cannot be minimised and all were classified as high risk of bias.

\section{Summary results of effectiveness}

ED returns: One low risk of bias $\mathrm{RCT}^{32}$ assessing an AECOPD discharge care bundle that incorporated individual components of a transitional care model failed to identify significant reductions in ED returns at 30 and 90 days compared with usual care.

Hospital re-admissions: A meta-analysis of four clinical trials of moderate-to-high risk of bias (figure 2) found that COPD discharge care bundles significantly reduced hospital re-admissions (RR: $0.80 ; 95 \%$ CI 0.65 to 0.99 ). Among BA studies, the per cent change (decrease) in 30-day hospital readmissions after bundle implementation relative to the period without discharge care bundle ranged from $-6.11 \%{ }^{37}$ to $-48.5 \%{ }^{28}$ (median per cent change: $-30.5 \%$; IQR: $-19 \%,-37.7 \%)$. Two BA studies with longer outcome follow-up had per cent decreases in hospital readmissions of $-42.4,{ }^{36}$ and $-30.1 \%{ }^{35}$ at 3 months. One BA study ${ }^{36}$ had a per cent decrease of $-50.7 \%$ in hospital readmissions at 1 year. The two ITS studies ${ }^{26}{ }^{31}$ reported conflicting results in annual hospitalisation trends before and after the implementation of the discharge bundles.

Secondary outcomes: Meta-analysis of four trials with moderate-to-high risk of bias (figure 3) failed to demonstrate that the implementation of discharge care bundles significantly reduce long-term mortality (RR: $0.74 ; 95 \%$ CI 0.43 to 1.28 ) in patients with AECOPD, with evidence of moderate heterogeneity $\left(\mathrm{I}^{2}=44 \%\right)$. Two trials of moderate-to-high risk of bias ${ }^{24} 30$ evaluated changes in QoL 6 months after discharge using the St George's Respiratory Questionnaire (SGRQ). Pooled MD in SGRQ scores (see online supplementary figure S2) failed to demonstrate significant differences between discharge care bundles and usual care 6 months after discharge $(\mathrm{MD}=1.84$; $95 \% \mathrm{CI}-2.13$ to 5.8 ). One RCT with moderate risk of bias failed to identify a significant effect of discharge care bundles on outpatient follow-up visits. ${ }^{25}$ Effects for patient satisfaction and economic outcomes were not reported in any of the studies included in the review. Due to the small number of RCTs included in the meta-analyses, a formal evaluation of subgroups and publication bias was not possible. ${ }^{23}$

\section{DISCUSSION}

Using high-quality methods including comprehensive search strategies to mitigate publication bias and efforts to reduce selection bias, this systematic review summarises evidence from 14 studies published in the scientific literature on the effectiveness of discharge care bundles in patients with AECOPD. The majority of studies in this area involve quality improvement initiatives with uncontrolled pre/post designs inherently accompanied by a high risk of bias. Meta-analysis of RCT data, however, showed that discharge care bundles after hospital admissions for AECOPD led to fewer readmissions with no significant improvements in mortality and QoL. The evidence from study designs of lower quality was congruent with these results.

The effectiveness of discharge care bundles to improve outcomes in patients with AECOPD is likely multifactorial, such as the number and characteristics of individual elements of care integrated in the bundle and the quality with which the bundle is implemented. The construct underlying care bundles is the value of incorporating a small number of individual interventions to ensure that evidence-based care is delivered consistently and reliably. ${ }^{11}$ We identified 26 distinct elements of care packaged in discharge bundles across individual studies in the review,

\begin{tabular}{|c|c|c|c|c|c|c|c|c|}
\hline \multirow{2}{*}{$\frac{\text { Study or Subgroup }}{\text { Linden } 2014}$} & \multicolumn{2}{|c|}{ COPD Discharge Bundle } & \multicolumn{2}{|c|}{ Usual Care } & Weight & $\begin{array}{c}\text { Risk Ratio } \\
\text { IV, Random, } 95 \% \mathrm{CI}\end{array}$ & \multicolumn{2}{|c|}{$\begin{array}{c}\text { Risk Ratio } \\
\text { IV, Random, 95\% CI }\end{array}$} \\
\hline & 4 & 124 & 15 & 131 & $17.9 \%$ & $0.28[0.10,0.83]$ & $\longrightarrow$ & \\
\hline Abad-Corpa 2013 & 7 & 56 & 17 & 87 & $25.4 \%$ & $0.64[0.28,1.44]$ & & - \\
\hline Lainscak 2013 & 11 & 118 & 13 & 135 & $27.2 \%$ & $0.97[0.45,2.08]$ & & \\
\hline Casas 2006 & 12 & 65 & 14 & 90 & $29.6 \%$ & $1.19[0.59,2.39]$ & & I \\
\hline Total $(95 \% \mathrm{CI})$ & & 363 & & 443 & $100.0 \%$ & $0.74[0.43,1.28]$ & & \\
\hline Total events & 34 & & 59 & & & & & \\
\hline $\begin{array}{l}\text { Heterogeneity: } \mathrm{Tau}^{2} \\
\text { Test for overall effect }\end{array}$ & $\begin{array}{l}0.14 ; \mathrm{Chi}^{2}=5 \\
=1.07(\mathrm{P}=\end{array}$ & $=3(P$ & $=0.15)$ & $1^{2}=$ & & & 0.01 Favours COPD Disch Bundle & $\begin{array}{cc}10 \\
\text { Favours Usual Care }\end{array}$ \\
\hline
\end{tabular}

Figure 3 Effectiveness of discharge care bundles on mortality for patients with an exacerbation of COPD (randomised controlled trials data only). 
with large differences in number of individual elements included in the bundles (eg, from 2 to 12 elements of care per bundle). The wide variety of individual elements of care included in bundles across the studies precludes drawing firm conclusions about which, and what combination of, individual interventions maximise the effectiveness of a discharge care bundle for COPD; however, some have suggested that adding too many elements may affect the reliability of the bundle as it becomes more and more difficult to implement. ${ }^{11}$

In reviewing the content of discharge care bundles in the individual studies, we found that the majority included a set of 'core' evidence-based interventions: demonstration of adequate inhaler technique, educational programmes on disease management, individually tailored care plans, assessment and referral for pulmonary rehabilitation, outpatient follow-up and referral to smoking cessation programmes. While the scientific evidence to support most of these individual interventions is relatively strong, ${ }^{38-40}$ some 'non-core' elements of care included in some discharge bundles lack the same level of strength in the underlying body of evidence. For example, there is very limited evidence of the benefit of breathing exercises in the comprehensive management of people with COPD. ${ }^{41}$ The majority of studies in this review did not clearly report how the lists of candidate interventions for the bundles were generated; therefore it is largely unknown how these interventions were selected and/or whether input from patients and clinicians was incorporated. The main challenge for the development of discharge care bundles for AECOPD, however, is not the identification of new effective individual interventions to incorporate in the bundles, but achieving balance between the number of elements included and the complexity of the intervention while preserving good consistency, fidelity and reliability in the implementation.

Overall, individual studies in the review seldom provided sufficient details about their implementation strategy, which translates into difficulties in integrating their findings within clinical practice. The majority of studies implemented the COPD discharge care bundles in respiratory wards of academic tertiary care centres, with less than half reporting the engagement of multidisciplinary clinical teams in the execution of the bundles. The diverse methodologies of studies using multidisciplinary teams for bundle implementation precluded a pooled analysis of the effectiveness on outcomes by this important implementation factor. Implementation strategies described in individual studies mainly involved education activities (outreaching, meetings and written materials), training of providers, and audit and feedback strategies. These findings are consistent with other reviews about implementation strategies of care bundles in hospitals. ${ }^{42} 43$

The reporting of implementation fidelity of COPD discharge care bundles in studies included in the review was very limited, with only five indicating the percentage of patients who received the individual bundle interventions. Implementation fidelity is critical to the successful translation of evidence-based interventions into practice ${ }^{18}$ and a mediator of the effectiveness of interventions ${ }^{44}$ particularly when interventions are complex and incorporate different providers along the continuum of care. There are several reasons why documenting implementation fidelity is critical to understanding the effectiveness of discharge care bundles in patients with AECOPD. Without clear and explicit information about the delivery setting, providers training, quality of delivery, programme differentiation and participants' responsiveness, ${ }^{44}$ it is possible that a Type III error (ie, poor implementation) may have accounted for the absence of detectable effects in some individual studies rather than a lack of bundle effectiveness. ${ }^{45}$ A clear reporting of implementation details and fidelity assessment in future studies will help to assess COPD discharge care bundle feasibility, reproducibility, applicability to a variety of settings and generalisability of the results. Individual studies in the review included all patients admitted to hospital for AECOPD and did not describe their study populations in terms of disease severity or baseline risk for future exacerbations. The evidence identified in this review was insufficient to draw any conclusions about whether implementation and effectiveness of COPD care bundles are determined by disease severity or other individual patient characteristics such as age, sex and socio-economic status.

Only three studies in the review reported that PCP were involved in the implementation of the bundles. Participation of PCP in COPD discharge care bundle implementation is key for the uptake of individual elements of care that, though initiated in acute/hospital care, are in fact typically executed in community settings (eg, pulmonary rehabilitation and smoking cessation programmes). Ensuring that clinical teams in both acute and outpatient settings understand and implement the core elements of the bundle is key for providing a seamless and consistent transition of care for patients with AECOPD discharged from hospital into the community.

At present, there is no consensus as to what comprises an optimal discharge care bundle for patients with AECOPD and which elements of care should be incorporated. Current research on discharge care bundles for AECOPD is limited and at moderate to high risk of bias. Results of this review should be interpreted with caution, as firm conclusions on the effectiveness of the bundles cannot be drawn. Differences in the content of bundles in studies included in the review highlight an urgent need for comparative effectiveness research to guide future implementation of discharge bundles in routine care of patients with COPD. Similarly, future synthesis of the evidence about strategies to reduce COPD hospital readmissions should assess the role of in-hospital admission bundles and the possible synergies associated with discharge bundles.

\section{Summary of results}

This systematic review found evidence suggesting that discharge care bundles are likely to reduce hospital re-admissions in patients with AECOPD. There is no evidence that these bundles reduce mortality or improve QoL.

\section{Strengths and limitations}

This is the first systematic review examining the scientific evidence about the effectiveness of discharge care bundles on re-admissions and other patient-oriented outcomes in patients with AECOPD. The strengths of this review pertain to the a priori protocol, comprehensive literature searches, the criteriabased selection of relevant evidence, the appraisal and reporting of risk of bias and the evidence-based inferences. Although the relatively small number of RCTs reporting comparable outcome measures precluded the assessment of publication bias, the exhaustive search strategy and inclusion of all trial types are likely to have identified most of the published and unpublished literature on the effectiveness of discharge care bundles in patients with AECOPD. The inclusion of a variety of study designs benefits evidence synthesis; they may be the best available information and may also answer questions related to longterm effectiveness, adverse events and rare events. Another important asset of the review is the control for multiple publication bias in the synthesis of results. We identified seven multiple publications, avoiding the inclusion of duplicate data, which 
may have skewed the evidence base in the analysis of individual study results. The present systematic review was limited to the analysis of the effectiveness of COPD discharge care bundles and did not evaluate the role that barriers and facilitators may play in bundle implementation. An examination of these factors is crucial and implies going beyond the experimental evidence to incorporate qualitative approaches. Finally, the risk of bias assessment was particularly challenged, due to incomplete reporting of methodological characteristics of the individual studies. This situation was particularly critical as four abstracts presented at international meetings were included in the review. We acknowledge that the abstract's conventional brevity may interfere with an accurate assessment of the study's risk of bias; however, we made efforts to contact authors of individual abstracts to obtain as much information as possible about their studies to guarantee a fair assessment of their methodological quality. The adoption of methods to reduce bias and enhance the transparency and reporting of primary studies in this field is needed to expand the evidence base of discharge care bundles for the management of COPD.

\section{CONCLUSIONS}

Discharge care bundles for patients with AECOPD can result in fewer hospital readmissions; however, the quality of the evidence in this field is weak and there are inconsistencies within the individual elements of the different COPD care bundles. No significant improvements were identified for mortality and QoL outcomes. Successful implementation of evidence-based discharge care bundles in patients with AECOPD requires consistent integration of best evidence into daily clinical decision-making. Evaluation of implementation fidelity of AECOPD discharge care bundles is key to understanding which elements of care are more likely to influence patient outcomes. Future studies should provide more granular details about bundle implementation and improve their methods to enhance the accuracy of estimates of effectiveness of discharge care bundles in patients with AECOPD.

\author{
Author affiliations \\ ${ }^{1}$ Alberta Health Services, Edmonton, Alberta, Canada \\ ${ }^{2}$ Department of Community Health Sciences, Cumming School of Medicine, \\ University of Calgary, Calgary, Alberta, Canada \\ ${ }^{3}$ Department of Emergency Medicine, Faculty of Medicine \& Dentistry, University of \\ Alberta, Edmonton, Alberta, Canada \\ ${ }^{4}$ School of Public Health, University of Alberta, Edmonton, Alberta, Canada \\ ${ }^{5}$ Division of Respirology, Cumming School of Medicine, University of Calgary, \\ Calgary, Alberta, Canada \\ ${ }^{6}$ Division of Pulmonary Medicine, Faculty of Medicine \& Dentistry, University of \\ Alberta, Edmonton, Alberta, Canada \\ ${ }^{7}$ G.F. MacDonald Centre for Lung Health, Covenant Health, Edmonton, Alberta, \\ Canada
}

Acknowledgements The authors thank Drs. Jean Bourbeau (Respiratory Epidemiology and Clinical Research Unit, Montreal Chest Institute, McGill University, Canada) and Annika Graham (Calderdale and Huddersfield NHS Foundation Trust, UK) for providing further information on their studies. They also thank Ms. Rachel Zhao (Alberta Health Services Knowledge Resource Service) for her assistance with the literature searches.

Contributors MBO, LD, RL, MB, BHR and MKS contributed to study conception and protocol of the review. MBO, KM and LD performed the systematic review. MBO performed the statistical analysis. All authors contributed to the interpretation of data. MBO drafted the paper and all authors provided critical revisions and contributed to the editing of the paper.

Funding This research was supported by Alberta Innovates Health Solutions (AlHS) through the Partnership for Research and Innovation in the Health System (PRIHS-2) programme. MKS is supported by a Heart and Stroke Foundation New Investigator Award. Dr. Rowe is supported by the Canadian Institutes of Health Research as a Tier I Canada Research Chair in Evidence-Based Emergency Medicine through the
Government of Canada. K. Mrklas is supported by an Alberta Health Services Post Secondary Education Tuition Award.

Competing interests None declared.

Provenance and peer review Not commissioned; externally peer reviewed. Data sharing statement List of excluded studies available from corresponding author.

\section{REFERENCES}

1 Ehteshami-Afshar S, FitzGerald JM, Doyle-Waters MM, et al. The global economic burden of asthma and chronic obstructive pulmonary disease. Int J Tuberc Lung Dis 2016:20:11-23.

2 Bourbeau J. Activities of life: the COPD patient. COPD 2009;6:192-200.

3 Wang Q, Bourbeau J. Outcomes and health-related quality of life following hospitalization for an acute exacerbation of COPD. Respirology 2005;10:334-40.

4 Global Strategy for the Diagnosis, Management and Prevention of COPD. Global Initiative for Chronic Obstructive Lung Disease (GOLD) 2016. http://www.goldcopd. org/ (accessed 20 May 2015).

5 Boulet LP, Bourbeau J, Skomro R, et al. Major care gaps in asthma, sleep and chronic obstructive pulmonary disease: a road map for knowledge translation. Can Respir J 2013;20:265-9.

6 Panella M, Marchisio S, Di Stanislao F. Reducing clinical variations with clinical pathways: do pathways work? Int J Qual Health Care 2003;15:509-21.

7 Kinsman L, Rotter T, James E, et al. What is a clinical pathway? Development of a definition to inform the debate. BMC Medicine 2010;8:31.

8 Middleton S, Barnett J, Reeves D. What is an integrated care pathway? Evid Based Med 2001:3:1-7

9 Resar R, Pronovost P, Haraden C, et al. Using a bundle approach to improve ventilator care processes and reduce ventilator-associated pneumonia. Jt Comm J Qual Saf 2005;31:243-8.

10 Lodewijckx C, Sermeus W, Panella M, et al. Impact of care pathways for in-hospital management of COPD exacerbation: a systematic review. Int I Nurs Stud 2011:48:1445-56.

11 Resar R, Griffin FA, Haraden C, et al. Using Care Bundles to Improve Health Care Quality. IHI Innovation Series White Paper. Cambridge, Massachusetts: Institute for Healthcare Improvement, 2012.

12 Lodewijckx C, Sermeus W, Vanhaecht K, et al. Inhospital management of COPD exacerbations: a systematic review of the literature with regard to adherence to international guidelines. J Eval Clin Pract 2009;15:1101-10.

13 Moher $D$, Liberati $A$, Tetzlaff J, et al. Preferred reporting items for systematic reviews and meta-analyses: the PRISMA statement. BMJ 2009:151:264-9.

14 Turner AM, Lim WS, Rodrigo C, et al. A care-bundles approach to improving standard of care in AECOPD admissions: results of a national project. Thorax 2015;70:992-4

15 Cochrane Effective Practice and Organisation of Care Review Group. Data collection checklist. http://epoc.cochrane.org/sites/epoc.cochrane.org/files/uploads/ datacollectionchecklist.pdf (accessed 20 May 2015).

16 National Heart Blood and Lung Institute. Quality Assessment Tool for Before-After (Pre-Post) Studies With No Control Group. 2014. http://www.nhlbi.nih.gov/ health-pro/guidelines/in-develop/cardiovascular-risk-reduction/tools/before-after. (accessed 24 Aug 2015).

17 Viswanathan M, Ansari MT, Berkman ND, et al. Assessing the Risk of Bias of Individual Studies in Systematic Reviews of Health Care Interventions. Assessing the Risk of Bias of Individual Studies in Systematic Reviews of Health Care Interventions. Agency for Healthcare Research and Quality Methods Guide for Comparative Effectiveness Reviews. March 2012. AHRQ Publication No. 12-EHC047-EF. http://www.effectivehealthcare.ahrq.gov/ (accessed 20 May 2015).

18 Carroll C, Patterson M, Wood S, et al. A conceptual framework for implementation fidelity. Implement Sci 2007;2:40.

19 Effective Practice and Organisation of Care (EPOC). EPOC taxonomy. Oslo: Norwegian Knowledge Centre for the Health Services, 2015. http://epoc.cochrane. org/sites/epoc.cochrane.org/files/uploads/2015\%20EPOC\%20Taxonomy\%20FINAL. pdf (accessed 15 Dec 2015).

20 Treadwell JR, Singh S, Talati R, et al. A framework for best evidence approaches can improve the transparency of systematic reviews. J Clin Epidemiol 2012;65:1159-62.

21 Deeks JJ, Altman DG, Bradburn MJ. Statistical methods for examining heterogeneity and combining results from several studies in meta-analysis. In: Egger M, Smith GD, Altman DG, eds. Systematic reviews in health care: meta-analysis in context. 3rd edn. London: BMJ Publishing Group, 2001:285-312.

22 Higgins JP, Thompson SG, Deeks JJ, et al. Measuring inconsistency in meta-analyses. BMJ 2003;327:557-60.

23 Sterne JA, Becker BJ, Egger M. The funnel plot. In: Rothstein HR, Sutton AJ, Borenstein M, eds. Publication bias in meta-analysis: prevention, assessment and adjustments. Chichester: Wiley, 2005:75-98. 
24 Abad-Corpa E, Royo-Morales T, Iniesta-Sanchez J, et al. Evaluation of the effectiveness of hospital discharge planning and follow-up in the primary care of patients with chronic obstructive pulmonary disease. J Clin Nurs 2013:22:669-80.

25 Casas A, Troosters T, Garcia-Aymerich J, et al. Integrated care prevents hospitalisations for exacerbations in COPD patients. Eur Respir J 2006;28:123-30.

26 Graham A. P290 Impact of a combined admission and discharge COPD care bundle on hospital mortality and readmission rates. Thorax 2012;67:A192-3.

27 Halpin DMG, Batten P, Chamberlain C. COPD discharge bundles: the Exeter experience 2011-12 [abstract]. Thorax 2012;67(Suppl 2):A:193.

28 Hopkinson NS, Englebretsen C, Cooley N, et al. Designing and implementing a COPD discharge care bundle. Thorax 2012;67:90-2.

29 Jennings JH, Thavarajah K, Mendez MP, et al. Predischarge bundle for patients with acute exacerbations of COPD to reduce readmissions and ED visits: a randomized controlled trial. Chest 2015;147:1227-34.

30 Lainscak M, Kadivec S, Kosnik M, et al. Discharge coordinator intervention prevents hospitalizations in patients with COPD: a randomized controlled trial. J Am Med Dir Assoc 2013;14:450.e1-e6.

31 Laverty AA, Elkin SL, Watt HC, et al. Impact of a COPD discharge care bundle on readmissions following admission with acute exacerbation: Interrupted time series analysis. PLOS ONE 2015;10:e0116187.

32 Linden A, Butterworth SW. A comprehensive hospital-based intervention to reduce readmissions for chronically ill patients: a randomized controlled trial. Am J Manag Care 2014;20:783-92.

33 Mann B, Ramhamadany E, Wilson S, et al. Assessing the impact of implementing a hospital discharge COPD care bundle on respiratory wards. Online J Clin Audits 2012;4. http://www.clinicalaudits.com/index.php/ojca/article/ viewArticle/233

34 Matthews $\mathrm{H}$, Tooley $\mathrm{C}$, Nicholls $\mathrm{C}$, et al. Care bundles reduce readmissions for COPD. Nurs Times 2013;109:18-20.
35 Seymour JM, Nedelcu D. The impact of a discharge care bundle on the 30-day readmission rate following hospitalisation for acute COPD exacerbation. Thorax 2014;69:A141-2.

36 Shorofsky M, Lebel M, Sedeno M, et al. Discharge care bundle for patients with acute exacerbations of COPD: benefit more likely to be seen beyond 30 days. Int J Respir Pulm Med 2015;2:024.

37 Yip NH, Brinson MD, Tylor BR, et al. Implementation of a discharge bundle to lower COPD readmissions [abstract]. American Thoracic Society International Conference 2012; 18-23 May 2012. San Francisco, CA http://www.atsjournals.org/ doi/abs/10.1164/ajrccm-conference.2012.185.1_MeetingAbstracts.A5146

38 McCarthy B, Casey D, Devane D, et al. Pulmonary rehabilitation for chronic obstructive pulmonary disease. Cochrane Database Syst Rev 2015;(2):CD003793.

39 Zwerink M, Brusse-Keizer M, van der Valk PD, et al. Self management for patients with chronic obstructive pulmonary disease. Cochrane Database Syst Rev 2014;(3): CD002990.

40 Godtfredsen NS, Lam TH, Hansel TT, et al. COPD-related morbidity and mortality after smoking cessation: status of the evidence. Eur Respir $J$ 2008;32:844-53.

41 Holland $A E$, Hill $C J$, Jones $A Y$, et al. Breathing exercises for chronic obstructive pulmonary disease. Cochrane Database Syst Rev 2012;(10):CD008250.

42 Borgert MJ, Goossens A, Dongelmans DA. What are effective strategies for the implementation of care bundles on ICUs: a systematic review. Implement Sci 2015;10:119.

43 Trogrlić Z, van der Jagt M, Bakker J, et al. A systematic review of implementation strategies for assessment, prevention, and management of ICU delirium and their effect on clinical outcomes. Crit Care 2015;19:157.

44 Durlak JA, DuPre EP. Implementation matters: a review of research on the influence of implementation on program outcomes and the factors affecting implementation. Am J Community Psychol 2008;41:327-50.

45 Dobson D, Cook TJ. Avoiding type III error in program evaluation: results from a field experiment. Eval Program Plann 1980;3:269-76. 